\title{
Confrontación epistemológica entre conductismo y psicología cognitiva, y sus repercusiones metodológicas *
}

\author{
M. T. ANGUERA \\ Universidad de Barcelona
}

$\oiiint$

Dirección de la autora: Universidad de Barcelona. Facultad de Psicología. Departamento de Psicología Experimental. Avda. de Chile, s/n. 08028 Barcelona.

Estudios de Psicologia, 1989, 37, 77-103 1985.

* II Jornadas de Psicología: Revisión de la Psicología del Aprendizaje. Oviedo, marzo 
La temática del aprendizaje es precisamente uno de los tópicos más adecuados, por no decir el que más, respecto al análisis de la trayectoria seguida en las últimas décadas por la ciencia psicológica y desde esta amplia perspectiva va a contemplarse.

Se ha repetido en innumerables ocasiones la existencia de contrastes entre puntos de vista y posiciones distintas, así como de una tendencia conciliadora manifestada en algunos casos, pero antes ¿podrían considerarse de forma diferenciada los planos epistemológico, metodológico y técnico/aplicado?, ¿qué tipo de relación se presenta entre ellos?, ¿en qué medida la problemática del aprendizaje se ha hecho eco de esta convergencia? Es evidente que existen muchos enfoques posibles respecto al abordaje del tema, cada uno de los cuales, a su vez, podría ser criticado a partir de las posturas que sustentaran los demás, aunque sólo fuera por la medida en que su contenido presentara límites altamente versátiles, o por los criterios de inclusión y exclusión. Plenamente conscientes de ello, vamos a tratar de perfilar el amplio panorama en el que nos movemos.

\section{EL APRENDIZAJE EN LA CONFRONTACION ENTRE CONDUCTISMO Y PSICOLOGIA COGNITIVA}

Si nos remontamos a los precursores del conductismo, los trabajos realizados en los primeros años de nuestro siglo sobre psicología del aprendizaje contribuyeron a generar una profunda insatisfacción con toda la psicología introspectiva, que juzgaban estéril o casi, y que iba a implicar una rebelión dirigida claramente contra toda la concepción tradicional de la psicología como análisis de los estados conscientes. El auge que había tomado la experimentación animal y el funcionalismo norteamericano (además de la influencia más lejana de las tradiciones filosóficas del objetivismo psicológico) condujeron claramente al conductismo, caracterizado cuirosamente por haberse tratado de un movimiento dotado de indudable empuje y gran vitalidad en su empeño por llevar a cabo un análisis riguroso y objetivo de los problemas de la psicología y que después terminó en fracaso.

De aquí entresacamos como importante punto de referencia lo que constituía para el conductismo «los problemas de la psicología»: ¿dónde tenían su punto de apoyo? La comunidad de conductistas creía en sí misma y su cohesión les llevó a estar convencidos de que eran poseedores de una tradición normal científica —en términos de Kuhn (1979)-, pero ello no era cierto, ya que la idea que compartían se reducía a una voluntad firme por el logro de la objetividad a toda costa y un énfasis sobre los problemas del aprendizaje, a pesar de que no tenían puntos de vista coincidentes; las diferentes teorías conductistas del aprendizaje tenían evidentemente un fondo común, pero se planteaban algunos interrogantes, tales como: ¿Qué es lo que se aprende?, ¿cómo se aprende?, ¿qué ocurre si se da el refuerzo? Estos y muchos otros temas controvertidos definieron las diferencias principales entre importantes teorías conductistas, determinando el curso y la dirección de la mayor parte de la investigación realizada durante este período. Cada una de las diferentes «tradiciones» - o, si se prefiere, «minitradiciones"- conductistas (por ejemplo, las de Hull, Tolman, Guthrie, Skinner, etc.) daba una respuesta a los interrogantes planteados a partir de 
su trabajo teórico y experimental, a la vez que trataba de rivalizar con las respuestas de sus oponentes conductistas, consideradas como erróneas; de aquí que, a pesar de los esfuerzos reales que se hicieron por rellenar los vacíos y grietas de un sistema teórico, éstos fueron insuficientes y, por tanto, incapaces para que las coincidencias entre ellos superaran a sus diferencias. Las primeras se reducian a la fe y defensa de la objetividad, un énfasis en la problemática del aprendizaje, y una voluntad para investigar indiferentemente estos problemas con sujetos humanos y animales, y de estas tres causas procedía toda la cohesión existente. El interés por la temática estudiada puede implicar una coincidencia conceptual en el punto de partida, pero no en el de llegada, lo cual es comprensible después de la respuesta diferencial que hemos reseñado respecto a ciertas preguntas básicas. Y por lo que se refiere a los otros dos elementos de cohesión, está claro que se trata únicamente de aspectos procedimentales, que podrían incluso considerarse como de principio, puesto que no detallan más acerca de las formas concretas de logro de tal objetividad rigurosa, o de las características de los sujetos experimentales.

En definitiva, ninguna escuela conductista podia progresar de forma extraordinaria, aunque porporcionara una respuesta acertada a sus inquietudes, dado que se partía de una «tabula rasa», y además, sus logros no eran tomados como plataforma de partida por otros; podría hablarse incluso de una cierta especialización de escuelas, y así la teoría de Tolman era la más apropiada para explicar los determinantes de la conducta en un punto concreto; la de Hull para relacionar la fuerza de respuesta al nivel del instinto y para las interrelaciones entre éstos en una situación experimental de una respuesta única; la de Guthrie para explicar los cambios en la conducta como una función de otros arbitrarios en la serie de estímulos; la de Skinner para controlar la topografía detallada de las respuestas aisladas de cualquier grado deseado de complejidad, etc. (Mackenzie, 1977).

¿Hasta qué punto era intencional esta carencia de un contenido definido en el conductismo? Se ha comentado repetidamente su fuerte compromiso metodológico y su convicción de que el uso riguroso de los procedimientos implícitos y formales de decisión - normas para llegar a cualquier tipo de conclusiones acerca de teorías, hipótesis y datos- garantizaría el desarrollo de un campo científico como un todo, así como la convergencia teórica de posiciones rivales dentro de ese campo, con lo cual las cuestiones metodológicas alcanzaban un lugar prioritario - básico- y las esenciales quedaban totalmente relegadas. Pero habría que reflexionar sobre cuáles eran las esenciales: en efecto, si consideramos el siguiente comentario de Koch (1964), resulta evidente la carencia de objetivos, que era tanto más acusada en la transición del conductismo clásico al neoconductismo, coincidente con el establecimiento y consolidación de la orientación positivista, que tuvo una resonancia absoluta en el campo de la psicología. Sus palabras son:

«El conductismo clásico ha sido un intento de escapar del estancamiento de las psicologías imperantes entonces al proporcionar a la psicología un procedimiento de decisión, que, según se esperaba, daria al movimiento un impulso inevitable hacia adelante. Pero aunque esta posición pronto alcanzó una hegemonía... degeneró con bastante rapidez en polémicas y en un deseo exagerado de 
hacer programas y más programas... Hacia el final de la década de 1920, había mucha experimentación "objetiva” pero pocas formulaciones de principios predictivos claramente establecidos en comparación con los excelentes logros de la física: sus teorias. Al contrario, la experimentación parecía sin finalidad fija, las hipótesis parecían "teóricas" y poco relacionadas con los datos, y el debate ineficaz e inútil.» (Koch, 1964, p. 9).

Sí había, sin embargo, un acuerdo inicial sobre algo esencial, que era el rechazo de la conciencia, y lo que ello implicaba en la propia definición de la conducta. Pero este rechazo no suponía -entendemos- ninguna afirmación respecto a lo sustancial y un movimiento o empresa surge y se consolida con mucha mayor garantía en base a un «slogan» o consigna o texto programático con afirmaciones a las que pretende dar vida, que con negaciones de lo que tiene que evitarse. Por esto, las figuras más representativas del conductismo partían de problemáticas específicas y muy concretas en el ámbito del aprendizaje, ya que faltaba la plataforma en que apoyarse.

Además, la confianza tradicional de la psicología en los conceptos de las ciencias físicas (otra prueba de la carencia indicada respecto a principios esenciales propios) suministró la base para una incorporación (más que un desarrollo autónomo) del positivismo, al ofrecer un modelo para el rechazo de todos los datos no comprobables, es decir, de los subjetivos, con lo que el «slogan» de rechazo de la conciencia se cumplía. La adopción de un modelo externo de objetividad por el que todas las entidades mentales podían ser rechazadas era consistente con el marco conceptual que el conductismo adquirió en su reacción a ellas y con el resultado obtenido de la psicología funcionalista comparativa, ya que el énfasis sobre el control ambiental de la conducta estimulaba una búsqueda de las causas que se podían observar, y no sobre las que solamente se podían proponer hipótesis (Mackenzie, 1977).

El principal efecto de la apelación sistemática al modelo externo de objetividad fue intensificar las propuestas metodológicas del conductismo y cuando antes nos preguntábamos sobre la posible intencionalidad respecto a la carencia de un contenido definido, era por el presentimiento de que los propios conductistas no tenían una idea clara acerca de cuál debía ser el objeto de la psicología (no vale el responder con una negación: «no a la conciencia...»), lo cual en parte puede ser debido por el efecto intrusionista de investigadores teóricos y empíricos de otras ciencias, que aniquilaron cualquier intento autónomo de avance en este sentido y además porque los propios conductistas estaban asombrados con su propio éxito, que podría considarse arrollador y en el que influyeron muchos elementos que los historiadores de la ciencia se han encargado de analizar; este mismo éxito parecía eximirles de algunas obligaciones, como la de argumentar adecuadamente sus bases o principios de partida, y por tanto la consistencia de lo que tenía que haber sido esencial y no secundario se habría tenido que asegurar por motivos de propia supervivencia. Dado además que una orientación positivista no implica un compromiso con la verdad de las ideas fundamentales de una teoría, pero sí con los fundamentos empíricos de ésta, se daban todos los ingredientes necesarios para que el compromiso metodológico compartido les ayudara a mantenerse teóricamente desunidos haciendo que sus diferentes posiciones fueran defendibles, en vez de unirlos más estrechamente para llegar a un acuerdo teórico básico. 
Analizado con la perspectiva y distanciamiento que da el paso del tiempo, es evidente que se trataba de un movimiento que tenía que sucumbir como tal, o al menos, debilitarse, dado que puede hablarse de la falta de un cuerpo doctrinal en sentido estricto y que es el que debía conferirle fortaleza en su esqueleto. Una vez que la rigurosidad en su despliegue metodológico -que consideramos por otra parte excelente y creemos valorarlo en su justa medida - había dado todo el fruto posible utilizando las comprobaciones de la conducta como la única fuente de datos, se habían creado unas expectativas cada vez más difíciles de acallar (lo cual constituye ya una interesante aportación que debe atribuirse al conductismo cuando se le haga un aséptico juicio crítico): todos los estudios realizados sobre diversos aspectos del aprendizaje resultaban insuficientes para un nuevo panorama de la psicología que trataba de revitalizarse mediante la adquisición de una identidad propia, no basada en aseveraciones negativas, sino positivas, como las más fructíferas.

La situación de crisis del conductismo era indudable y a partir de 1950 se había enfrentado cada vez con más dificultades conceptuales, metodológicas y empíricas. Las anomalías no son el único factor que conduce al cambio, sino que -más importante es el surgimiento de nuevas teorías, pero éstas no aparecen sin que antes se hayan hecho necesarias (Gabucio, 1983, p. 70). Existen diversas relaciones de índices de la decadencia conductista (Mackenzie, 1972; Palermo, 1971) y dado que el aprendizaje se había constituido en tópico central, la consecuencia inevitable era que sobre él iban a converger resultados «disidentes»: así, se pusieron de manifiesto anomalías en conductas de transposición al entrenar a niños en la elección del mayor estímulo en tareas de aprendizaje de discriminación (Spence, 1937), o, en el mejor de los casos, se producía una relajación de los principios conductistas (Kendler \& Kendler, 1962); imposibilidad de demostrar la interferencia mediada en aprendizaje verbal (Bugelski y Sharlock, 1952); controversia sobre el aprendizaje en un solo ensayo (Rock, 1957; Estes, Hopkins y Crothers, 1960); estudios sobre el imprinting (Hess, 1959); polémica sobre el uso teórico de variables intervinientes y constructos hipotéticos (MacCorquedale \& Meehl, 1948), y muy especialmente, entre otros, la polémica producida por los trabajos de Chomsky $(1957,1959)$, especialmente en su crítica a Skinner (1957), además de una serie de investigaciones llevadas a cabo en torno a 1950 cuya característica común fue la de violar las expectativas del postulado hulliano de reducción del impulso (Caparrós, 1979), y que ponían de manifiesto la absoluta imposibilidad de explicar el aprendizaje mediante dicho principio.

Contemplado desde la evolución del conductismo, nos hallamos entre la era de las teorías y la fase de las crisis (Yela, 1980). Si hasta ahora se había destacado la relativa continuidad metodológica y la unidad sólo formal, ni Watșon y los neoconductistas comparten un cuerpo común de conocimientos, explicaciones y resultados fundamentales. Las diferencias entre los neoconductistas aún son mayores respecto al contenido. Todos estudian el aprendizaje animal en parecidas situaciones de laboratorio, pero incluso en este simple contexto discrepan en todo (Yela, 1980). Respecto a los interrogantes antes planteados discrepan en lo que el animal aprende (respuestas, conexiones $\mathrm{E}-\mathrm{R}$, asociaciones entre estímulos $\mathrm{E}-\mathrm{E}$, expectativas, relaciones); en el mecanismo por el que el animal aprende (contigüidad, reforzamiento, 
ensayo y error vicario, confirmación de expectativas, transposición), y en la interpretación de ese mecanismo (muestreo de estímulos y respuestas en el establecimiento incremental de conexiones entre los patrones de energias y de movimientos, o refuerzo como reducción de necesidades, reducción de impulsos, satisfacción hedónica, mantenimiento de la propia actividad, cambio significativo en la estructura de la estimulación o mera comprobación empírica del aumento de la probabilidad de respuesta).

Es lógico entender que la psicología, que como ciencia había crecido enormemente en sofisticación técnica, estaba necesitada de un esquema que le permitiera una estructura coherente y los pasos que hacia aqui se dirigían pasaban por la internalización de la información de cada sujeto acerca de su mundo externo (Boneau, 1974), iniciándose con ello un desplazamiento basculante en el concepto de la psicología.

Se había producido un cambio en tal objeto de estudio desde lo externo a los procesos internos inferidos, aceptándose unidades (como cognición, plan, mediador, o esquema) que consolidarían la nueva orientación cognitiva, en donde los datos y los métodos daban un papel relevante al sujeto humano (de momento sólo a él) (Anguera, 1984) a diferencia del conductismo (Breger, 1969), en donde - y a pesar de la opinión de algunos autores, como White (1876)- es cuestionable el papel activo del sujeto, sometido a un claro mecanicismo.

Es decir, mientras que el conductismo radical (Day, 1977) sostuvo que las acciones del hombre y, por tanto, el aprendizaje, se debían explicar sólo en términos de variables observables, sin ninguan vicisitud interna, y posteriormente los propios teóricos del $\mathrm{E}-\mathrm{R}$ inventaban mecanismos hipotéticos sin remordimientos (Tous, 1978), ahora, la razón fundamental para el estudio de los procesos cognoscitivos es, simplemente, "porque están ahí» (Neisser, 1979, p. 16), y por tanto se convierten en obligado centro de interés de la psicología, que deberá estudiar los procesos mentales implicados en la adquisición, procesamiento y utilización del conocimiento o información. La base de esta formulación está, pues, en que el sujeto internaliza, es decir, extrae información acerca del mundo exterior y sus relaciones con él, construyendo un análogo interno de posiciones críticas de esta información (Boneau, 1974); pero para calibrarlo en su justa medida (Atkinson, 1977) era necesario conocer su ámbito, presupuestos, metodología, o su relación con las restantes orientaciones de la psicología, es decir, en una palabra, concretar el «referente» (Mayor, 1980, p. 214) de la psicología cognitiva, pues de lo contrario,

«contemplada la cuestión desde esta perspectiva, la situcación es realmente confusa, pues la psicología cognitiva queda convertida en un inmenso cajón de sastre donde cabe todo lo que de alguna manera tenga relación con el conocimiento» (Pinillos, 1980, p. 275).

Kendler (1981), importante analista crítico de la psicología a nivel epistemológico, afirma que las suposiciones centrales de la psicología cognitiva son difíciles de identificar debido a las influencias de las diversas tradiciones y ciencias que se sitúan alrededor (lingüística, computación, teoría de la información, etc.), pero podrían concretarse en que los sujetos adquieren representaciones internas (información) del mundo físico que pueden 
transformarse en diversos estadios o niveles, implicándose diferentes planes o estrategias (Miller, Galanter y Pribram, 1960) en la resolución de problemas para utilizar correctamente la información válida, y el rendimiento es una función conjunta de los principios de la cognición y de la acción. Esta gran flexibilidad del paradigma de procesamiento de la información no sólo iba a tener repercusiones a nivel metodológico (Seoane, 1979), sino también a nivel conceptual, ya que la ausencia de una meta teórica "restrictiva" que favoreciera una cierta circunscripción del trabajo empírico llevó a una muy amplia diversidad de contenido, en donde prácticamente cabe cualquier aspecto que consideremos del comportamiento (Mayor, 1984), pudiéndose entender, por exclusión, que toda esta serie de líneas de trabajo generadas al amparo de la corriente cognitiva se caracterizan por dos puntos esenciales (Zacaggnini y Delclaux, 1982), que son el rechazo explícito o implícito del reduccionismo conductista y la necesidad de contar con el organismo y con su capacidad de "elaborar" la información del medio de forma que se alcancen niveles superiores.

Ahora bien, dado que existe un amplio reconocimiento de que la psicología cognitiva estudia básicamente la percepción, la memoria, la atención, el reconocimiento de formas, la solución de problemas, la psicología del lenguaje y el desarrollo cognitivo (Neisser, 1981) y, dada la identificación, en palabras de Wickelgren (1979), entre psicología experimental y psicología cognitiva, ¿dónde ha quedado relegado el aprendizaje, si tenemos en cuenta que los principales tópicos presentes en la psicología experimental clásica son tiempos de reacción, psicofísica, emoción, sensaciones, percepción, memoria y, especialmente, aprendizaje?

A pesar del aprovechamiento en algún caso de viejos tópicos (Hilgard, 1980), aparece un «remozamiento lexicológico" de la mano del giro conceptual (Arnau, 1982), en el que, por otra parte, se aprecia una falta general de coincidencia, al menos en la utilización de las «funciones cognitivas" (Isaacson, Hutt y Blum, 1965), o "procesos cognitivos» (Whittaker, 1965; Kendler, 1968; Hilgard y Atkinson, 1967; Ruch, 1963), tal como señala Moroz (1972). Luego, no sólo se esfuma el conductismo, sino que deja de tener sentido e identidad como tal el que había sido su tópico estrella. Interesaba ahora, por pura coherencia interna, el estudio de los parámetros más complejos y relevantes de la conducta humana y además, por parte de algunos psicólogos cognitivos, se plantea la necesidad de que la psicología animal se reoriente a estudios que presenten la posibilidad de generalizar conocimientos a humanos, tanto en los procesos automático-asociativos como los controlado-cognitivos (Veá, 1984), existiendo una continuidad entre las conductas de aprendizaje más simples (Díez-Chamizo, Blackam y Thomas, 1981) y la resolución de problemas más complejos (Veá, 1983).

En los últimos veinticinco años no se ha realizado ningún esfuerzo importante para presentar un armazón teórico que explicara todo el aprendizaje, salvo la obra de Mowrer (1960a, 1960b), que ha recibido por lo demás escasa atención. El análisis conceptual parece haber sido abandonado desde los últimos grandes teóricos, como Hull, Spence, Guthrie y Tolman. Los problemas de aprendizaje, que quedaron después en manos de Skinner y sus seguidores como grupo de influencia mayoritaria, desarrollaron lo que se denomina «aprendizaje verbal» (Bugelski, 1980), que es un exponente en sí mismo del cambio producido y así se ha pasado de la preocu- 
pación por listas de palabras reales en lugar de sílabas sin sentido, desplazándose el interés básico del learning a la performance, y los sujetos ya no aprenden los ítems de la lista, sino que los conocen, con lo que la adquisición fue desenfatizada (Lachman, Lachman y Butterfield, 1979) mientras ocurría lo contrario con la recuperación; esto no quiere decir que de forma radical y absoluta el aprendizaje fuera olvidado -y así en el procesamiento de información de recuerdo libre todavía permanecen vestigios, como la inclusión por Anderson (1972) de un componente de aprendizaje-, pero en cualquier caso la concepción era totalmente distinta, como por otra parte se puede comprobar en sus inicios si recordamos la controversia sobre las causas del olvido que llevaron a cabo Peterson y Peterson (1959).

Los teóricos del aprendizaje afirman actualmente que el aprendizaje en tanto que resultado es considerado en términos de estructuras internas en las que de alguna forma se representan tanto las relaciones observadas entre distintos elementos del ambiente como las características de los elementos mismos que intervienen en la relación (Dickinson, 1980), así como el hecho de que estas estructuras internas hayan sido caracterizadas en diversas formulaciones alternativas a los principios tradicionales del reforzamiento y la conexión E-R, como expectativas (Bolles, 1972), organizaciones de contingencias (Bindra, 1974, 1978), o unidades situación-acto-resultado (Boneau, 1974), y que mantienen diferentes semejanzas con Toman (1932).

En cualquier caso, a pesar de la concepción general del aprendizaje expresada por Boneau (1974, p. 298), de que «la conducta está determinada fundamentalmente por eventos internos al organismo y posibilita que la misma se base en procesos cognitivos de diversa índole», los investigadores en psicología cognitiva ya estaban mucho más interesados por la memoria y la resolución de problemas (Houston, 1981) que por el aprendizaje y habían descubierto la importancia del significado, puesto que habian abandonado el concepto neoconductista de asociación como una conexión E-R (Wilson, 1980); no querían limitarse a la consideración de eventos estrictamente observables, sino el famoso look inside the head. Había cambiado, pues, la naturaleza de los problemas por los que se preguntaban y la forma de trabajar en ellos.

\section{REPERCUSIONES METODOLOGICAS}

Mientras Hull (1943, 1951), algunas décadas anteriores, argumentaba que para mantener el rigor debía evitarse la consideración de eventos mentales (mentalistic events), los modernos psicólogos cognitivos estaban convencidos de que, después de todo, hay que comprender los complejos eventos mentales aunque no puedan ser observados directamente y que, si Hull y los conductistas habían sido rigurosos en su época, las nuevas técnicas experimentales y los instrumentos analíticos con que se empezaba a disponer de forma creciente serían suficientes para el planteamiento y respuesta de nuevas cuestiones ahora candentes y entre ellas estaba pendiente por supuesto, el cambio de conceptualización del aprendizaje.

El replanteamiento epistemológico de la psicología no podía afectar solamente a la dimensión de los datos básicos o al modelo explicativo que los integraba (Arnau, 1982), teniendo en cuenta la naturaleza polisemántica del 
término "explicación" (Fierro, 1982), sino que afectaba muy estrechamente a los problemas de método, tanto por la necesidad de adecuación de éste a la materia de estudio, como por la incidencia del abandono del empirismo lógico (Seoane, 1980).

El problema no se planteaba tanto a raíz de la vía o enfoque hipotético-deductivo, muy debatido en la polémica Popper-Kuhn (Popper, 1963; Kuhn, 1979), sino que apareció primeramente en las técnicas experimentales, entonces consideradas por muchos como el punto de unión de la psicología con las ciencias naturales. Y si tenemos en cuenta que:

«la nueva "perspectiva" del procesamiento de la información rompió el vínculo E-R que había marcado la investigación psicológica durante muchos años y trasladó el campo de interés desde lo exclusivamente observable hasta los procesos mentales humanos que no pueden ser sino inferibles" (Delclaux, 1982, p. 29).

se abre una amplia panorámica en cuanto a las repercusiones metodológicas que iban a hacerse patentes desde entonces, tanto por las ciencias más o menos afines que le hubieran influido desde el exterior, como la ingeniería humana y de las comunicaciones, la ciencia de los computadores y la lingüística (Lachman, Lachman y Butterfield, 1979), como, en muy importante medida, aunque quizá menos comentado, por la adquisición de una perspectiva sistémica en el estudio del sujeto, que ha llevado a considerar que no es la conducta el verdadero objeto de la psicología sino la conciencia -entendida primeramente como medio para estudiar la conducta (Yela, 1974)-, o el conocimiento (Seoane, 1982), o la actividad (Mayor, 1984a), o tanto otros (Cruz Hernández, 1982) con debates cuyos resultados son los supuestos básicos teóricos y metodológicos que van a configurar el marco de referencia posibilitador de la investigación empírica de las comunidades psicológicas reestructuradas en ese mismo proceso de recuperación de identidad científica (Caparrós, 1984). Pero se trata de un marco de referencia plural y en donde el conformismo sería señal evidente de incapacidad por superar los graves problemas que implica el viraje epistemológico.

En efecto, nos hallamos, desde la emergencia del cognitivismo, ante una auténtica legión de publicaciones (Forrester, 1984) que sólo en parte son coincidentes en cuanto al carácter de paradigma que pudiera o no tener (Briskman, 1972; De Mey, 1982; Zacaggnini y Delclaux, 1982; Caparrós y Gabucio, 1984; Mayor, 1984b) —en cuyo debate aquí no entraremos-, y es cierto que existen muchas cuestiones pendientes que plantean serias lagunas sobre cómo la psicología cognitiva trata de reconstruir mediante modelos verificables todos aquellos procesos mediante los cuales el input sensorial es transformado en información, reducido, elaborado, almacenado, recuperado y utilizado. Dada la asimilación afirmada por varios autores entre psicología experimental y psicología cognitiva (Massaro, 1975; Wickelgren, 1979) y a la que anteriormente nos hemos referido, la primera ya no participa de las características de objetivismo metodológico de un conductismo carente de contenido definido, sino que a través de la elaboración de modelos verificables y de simuladores, con nuevas técnicas como leit motive, nos presenta unas sendas altamente sugerentes de momento, que han 
actuado - y aún actúan- como una especie de marca de calidad con suspense del enfoque y/o paradigma del procesamiento de la información.

Por lo que se refiere a la metodología, en parte de los casos se recurre a un mismo tipo de investigación manipulativa-experimental, por lo que, hasta un cierto punto, no podría hablarse de un choque de metodologías (Arnau, 1982), habiéndose seguido generando, en consecuencia, los mismos errores y sesgos propios de tal metodología en la época conductista, reflejados en las múltiples críticas y dudas sobre la utilidad y validez de la experimentación (Gadlin y Ingle, 1975; Kruglanski, 1976; Alvira, Avia, Calvo y Morales, 1979), así como en el acuñamiento de conceptos para caracterizar las diversas distorsiones que afectan a la situación experimental, como el efecto de experimentación de Rosenthal (1964), las características de la demanda de Orne $(1962,1969)$, las limitaciones del conocimiento experimental de Harré y Secord (1972), o los límites de la autorreflexión experimental de Gadlin y Ingle (1975), por citar los más conocidos, dejando ya los problemas de la ética de la experimentación o el alcance de las aplicaciones del conocimiento experimental (Mayor, 1982), o en general, el efecto de investigador (Barber, 1976).

Sin embargo, no todo es idéntico ni nuevo en sentido absoluto (Anguera, 1983a) pues, por ejemplo, la técnica más frecuentemente empleada en el laboratorio de Wundt era la medida de los tiempos de reacción, con la adopción directa del programa sugerido por Donders (1868-1869), llevándose a cabo inferencias acerca de las capacidades de procesamiento de información humanas sobre la base de tiempos de respuesta medidos bajo condiciones que variaban sistemáticamente. $Y$ en la actualidad, cumplido con creces un siglo desde que Wundt fundara su famoso laboratorio en Leipzig, esta misma temática —estudio de los tiempos de reacción- tiene plena vigencia y no sólo ello, sino que algunos autores del prestigio de Sternberg (1969a, 1969b, 1975), aunque señalen las dificultades del método sustractivo de Donders, con sus estudios de exploración atentativa en memoria inmediata, están revalorizando dicho trabajo, con el cual comparan sus propios resultados, siendo indudable el considerable interés que despertó la exploración de las diferencias individuales en el tiempo de reacción y el estudio de las implicaciones de la cronometría mental en las investigaciones psicofísicas (Posner, 1978). No hay duda, en efecto, de que aunque en la actualidad se considere el problema como más complejo tuvo mérito su análisis original (Lachman, Lachman y Butterfield, 1979) y únicamente se haya tratado de extender y refinar la técnica (Taylor, 1976; Alto, 1984).

Luego, si en este campo de estudio ha pervivido (o al menos resurgido) el interés por los tiempos de reacción, ¿cómo ha ocurrido lo contrario con la temática del aprendizaje desde el esplendor del conductismo hasta nuevos días? Hemos visto como, en contraste con el enfoque conductista, el de procesamiento de la información puede ser caracterizado como el estudio de procesos que no pueden ser directamente observados. Es indiscutible, según la concepción moderna de la ciencia (Weimer, 1979), que pueden hacerse inferencias válidas sobre procesos mentales no observables estudiando la conducta que resulta de ellos o que depende de su actividad. No obstante, estos procedimientos «indirectos» no siempre son útiles en situaciones donde un sujeto no comete errores o donde las condiciones experimentales no han degradado drásticamente la ejecución (Ato, 1984). En 
el caso del tiempo de reacción, podrian aducirse que la única propiedad de los procesos mentales que puede estudiarse directamente en el organismo intacto, y mientras se encuentra realizando cualquier actividad, es su duración.

En el ámbito del aprendizaje, puede afirmarse que su exclusión de los manuales es prácticamente general, indentificándosele con memoria, y hablando de «aprendizaje de palabras», cuando en realidad es «memorización de palabras»; en los estudios de memoria semántica y en los de formación de conceptos se trata el tema de la adquisición de nueva formación, pero raramente se lo califica de aprendizaje. En realidad, son muchos los autores que consideran que la memoria es la clave de todos los procesos cognitivos (Zaccagnini y Delclaux, 1982), y los propios Norman y Rumelhart afirman:

«En nuestras investigaciones sobre el lenguaje, percepción visual, solución de problemas y aprendizaje, llegamos a la conclusión de que el aspecto que los relaciona entre sí es el concepto unificador de cómo se representa la información en la memoria» (Norman y Rumelhart, 1975, p. 3).

El aprendizaje parece difícil de tratar desde este enfoque, aunque en el caso de sujetos animales se esté trabajando intensamente (Rajecki, 1983; Catania, 1984; Premack, 1984; Roitblat, 1984; Staddon, 1984; Vea, 1984), especialmente en mapeo cognitivo como conocimiento y representación del entorno (Downs y Stea, 1977), con un título de capítulo sumamente indicativo: Learning: How environments get mapped (Downs, 1981), estudiándose el aprendizaje de tareas, por ejemplo, en laberintos con pistas propias o internas -características visibles y diferenciadas del laberinto que hacen que sus distintas partes sean distintivas, como color o textura del suelo y pistas externas o exteriores al laberinto, pero en base a las cuales los sujetos pueden aprender igualmente una tarea de discriminación, y siendo la medida del aprendizaje el porcentaje de respuestas correctas (Diez-Chamizo, Sterio y Mackintosh, en prensa). Aquí podría afirmarse que casi todas las variables dependientes al uso en psicología se relacionan arbitrariamente con el constructo del cual son una medida; por ello, un investigador interesado en la cantidad de aprendizaje que puede lograrse por alguna manipulación experimental podría tomar el porcentaje de respuestas correctas en un postest como variable dependiente, en cuyo caso el porcentaje correcto no es sino un sustituto de la variable real de interés (cantidad de aprendizaje) (Ato, 1984).

Son muchas las reflexiones que de aquí pueden derivarse (Anguera, 1983b), y se nos sugiere que, si bien es cierto que antes -época conductista- el experimento había sido la herramienta metodológica más utiliza$\mathrm{da}$, también lo es que Wundt no pudo solucionar muchos problemas por esta vía, aun si salvamos la distancia de un siglo de forma tolerante por el limitado bagaje de recursos procedimentales de aquel entonces. Wundt era muy consciente de las estrecheces de su psicología experimental, y sabía que éstas se hallaban íntimamente relacionadas con el tipo de experimento que propugnaba, y precisamente

«el error de Wundt consistió en mantener su experimento como el único válido en pisocología. Ya en su tiempo, muchos entendieron que incluso en una 
psicología introspectiva de la conciencia son posibles otros tipos de experimentos» (Caparrós, 1984, p. 121).

En este punto, sería interesante preguntarnos tanto por estas posibles alternativas, como por la necesidad "vital» de mantenerse en unos cauces metodológicos tradicionales. Sobre ello, es ilustrativo tener en cuenta que

«las estrecheces de aquella psicología experimental fueron notadas de inmediato. Pero Wundt consiguió demostrar en su laboratorio que aún con sus herramientas era posible el plantear unos problemas psicológicos resolubles y proporcionar unos instrumentos conceptuales y metodológicos para su resolución y formulación. Al parecer, en el marco general de la ciencia de entonces, esa actividad experimental era un requisito necesario para que la psicología adquiriera identidad científica diferenciada» (Caparrós, 1984, pp. 121-122).

No pretendemos llegar al simplismo reduccionista de asimilar la introspección con el rechazo del experimento, lo cual sería absolutamente falso y desmesurado, pero sí su insuficiencia para cubrir las exigencias metodológicas planteadas. Lo prueba el hecho de que el intento de aunar -un tanto a la fuerza - el rigor metodológico con el tratamiento de problemas "cognitivos" ha fallado por dos puntos cruciales (Zaccagnini y Delclaux, 1982): Por un lado, no se ha conseguido romper con el reduccionismo heredado de la época conductista, con lo que implica de operativización y control de variables que fuerzan la obtención de puntajes - por otra parte, previos e imprescindibles para la metodología experimental-, y por otro lado, los diferentes autores renunciaron a buscar y elaborar teorías generales, quedándose en miniteorías parceladas y sumamente específicas, y en muchos casos tan solo en micromodelos que se intercalan entre el input y el output con la pretensión de buscar explicaciones ad boc de muy diferentes tipos (Gergen y Gergen, 1982) para cada pequeño problema con independencia de los demás, por lo que nos hallamos con profusión de pequeños conjuntos de datos que no pueden relacionarse de forma coherente, sino que, por el contrario, se acumulan sucesivamente (Newell, 1973) sin perspectivas de solución a partir de un modelo aglutinante. Este procedimiento -método funcional- que lleva a postular micromodelos (generalmente inspirados o copiados de la ciencia de las computadoras) para comprobarlos mediante pequeñas series de experimentos causales cubren una parte -amplia cuantitativamente en razón de su profusión, pero pobre desde el punto de vista cualitativo- que además se halla afectada por la falta de validez ecológica que está incidiendo sobre buena parte de los estudios de laboratorio. Es evidente que si se renunció a buscar modelos generales era más por la dificultad que planteaba que por un mero capricho de la parcelación del campo de estudio, a lo que se añadía, además, la posible distorsión de la validez interna del experimento (Arnau, 1979) debido a la contraposición entre modelos de sujeto (Seoane, 1980), por lo que la experimentación no pondría de manifiesto ninguna relación o característica original de los datos, sino que se limitaría a explicitar y desarrollar el modelo que sirve de punto de partida al investigador, hasta que otro modelo se contrapusiera al anterior, restando así validez interna al diseño empleado.

Luego podríamos atisbar que la confrontación epistemológica entre conductismo y psicología cognitiva sí implicaba unas repercusiones meto- 
dológicas de amplia angular, dado que desaparecía la condición de observabilidad propia del reduccionismo conductista y adquirían mejor rango los contenidos accesibles a la conciencia; el verdadero problema se hallaba en el impasse de búsqueda de nuevas metodologías más eficaces -análisis introspectivo, ventana abierta (Eysenck, 1984), etc.-, en muchos casos procedentes de otras ciencias que estaban en aquel momento incorporándose dentro de la ancha cobertura de la emergencia cognitiva, y cuyo ejemplo incuestionable es la metáfora o analogía del ordenador (De Vega, 1982), extraordinariamente fértil por lo que ha significado de herramienta conceptual y fuente de hipótesis como de facilitador de sistemas notacionales útiles para formalizar modelos. La tarea del teórico ya no será, a partir de ahora, descubrir una serie de ecuaciones matemáticas que encajen con los índices probabilísticos de un conjunto determinado de datos empíricos, sino que, por el contrario, se tratará de describir de forma explícita un conjunto de reglas, operaciones o "programas» que controlan la actividad cognitiva.

La simulación (Zeigler, Elzas, Klir y Oren, 1979), cuyo interés fundamental reside en la elaboración de programas que constituyan una buena teoría psicológica (formalización psicológicamente plausible) - simulación de imágenes mentales de Kosslyn y Schwartz (1977), simulación de solución de problemas de Newell y Simon (1972), simulación de tareas de seriación (Baylor, Gascon, Lemoyne y Pothier, 1973), simulación de tareas de inclusión de clases (Klahr y Wallace, 1976), etc.-, y la inteligencia artificial, cuyo objetivo consiste en el funcionamiento satisfactorio de los programas (Cercone y McCalla, 1984), sin que actúen aquí las constricciones empíricas y teóricas propias de la simulación, y por tanto sin pretender la reproducción de todos los parámetros relevantes de la actividad humana - con lo que desaparecería el reduccionismo antes criticado (Dennett, 1979)-, que se convierten en las dos grandes «salidas" metodológicas ampliamente expandidas a lo largo de los últimos años.

Teniendo en cuenta que la analogía mente-ordenador es funcional, no física, la similitud entre ambos es obvia, y ha sido explotada en ambas direcciones, ya que por una parte presta a los psicólogos un vocabulario, unas directrices y una instrumentación adecuadas para el estudio de la mente, y por otra los ingenieros electrónicos (Aiken, Babbage, von Neuman, Shannon, Turing, Walter er al., 1975) y los técnicos en inteligencia artificial tratan de trasladar intuitivamente sus ideas sobre el funcionamiento mental al campo del ordenador (De Vega, 1984); además, los psicólogos cognitivos toman como modelo el ordenador para plantear hipótesis psicológicas y elaborar interpretaciones teóricas. No en vano la mente y el ordenador son sistemas de procedimiento que codifican, retienen y operan con simbolos y representaciones internas, aunque existen aspectos «cualitativos» de la informacón que no son programables (Fodor, 1981), y también es cierto que el orden de complejidad con el que puede tratar un sistema exclusivamente discreto (como el ordenador) está prefijado, y su versatilidad depende de la intervención de un agente externo capaz de realizar una codificación discreta adecuada de las reglas y procedimientos necesarios para tratar problemas nuevos (Rivière, 1984). No es tampoco, en consecuencia, suficiente...

Antes mencionábamos el carácter demarcador de la introspección por lo que se refiere a las insuficiencias metodológicas en el trabajo wundtiano, y 
lo cierto es que actualmente el tema de la introspección se ha replanteado como consecuencia del papel dominante de la psicología cognitiva, dando lugar a posicionamientos contrapuestos debido a que los aspectos experienciales y conscientes de la actividad mental son objeto lícito de estudio, pero con el «handicap» de que la experiencia consciente no proporciona datos fiables sobre los fenómenos mentales (De Vega, 1984).

Aquí podríamos parafrasear unas palabras interesantes a este respecto:

«La historia de la ciencia enseña que, cuando una teoría que tiene éxito entra en conflicto con un escrúpulo metodológico, por lo general es el escrúpulo el que cede"... «Quizá no haya mejor forma de establecer qué está metodológicamente permitido en una ciencia que la de investigar qué precisa una ciencia que tiene éxito" (Fodor, 1981, p. 75).

Y lo que se precisa en psicología cognitiva es la comprensión detallada de los mecanismos y estructura interna de los procesos cognitivos; por tanto es lógico que haya resurgido el interés por arbitrar procedimientos que permiten incrementar la densidad temporal de las observaciones de forma que revelen estados intermedios del proceso al ser cuidadosamente analizadas, y uno de los medios factibles de posibilitarlo son los «reports» verbales, que ya habíâ sido sospechosos como datos cuando unas décadas antes se produjo el triunfo del conductismo sobre el introspeccionismo entonces imperante, y Watson (1913), en su influyente trabajo Psychology as the Behaviorist views it, lanzaba un ataque frontal al estudio de la conciencia, y criticaba el método introspectivo y sus resultados.

Esta crítica, sin embargo - y esta distinción es importante-, no se dirigía contra todos los usos de los informes verbales como datos, sino específicamente contra los métodos analíticos y resultados de los introspeccionistas clásicos. Cuando Watson (1913) señalaba la falta de reproductibilidad de introspecciones analíticas a partir de diferentes laboratorios, lo que se estaba planteando era la problemática relativa a la comunicabilidad del significado, preguntándose hasta qué punto podemos estar seguros de que un observador que «introspecciona» utiliza el lenguaje de la misma forma que un experimentador que «interpreta» (se entiende datos objetivos). Analicemos el siguiente fragmento:

"Después de haber llevado a cabo un análisis de búsqueda sobre lo que es propio que realicen varios jugadores de golf, ¿qué quedaría de los propios registros de los sujetos? Supongamos de nuevo que analizamos sus respuestas a las preguntas que les formulamos y las incorporamos a nuestro objetivo. Tienen relativamente escaso valor. Ninguna, puesto que los estudios objetivos sobre el golf han sido hechos confiando en el report verbal de un jugador de golf. El dirá que nunca pierde de vista el balón cuando da un golpe. La cámara muestra que es un mentiroso" (Watson, 1920, p. 100).

Lo que se cuestiona en este fragmento no se refiere a la memoria del sujeto respecto a un instante o ejemplo específico, sino como él piensa que produce su actividad cuando se le pregunta sobre ella. Watson hacía una clara distinción entre la introspección analítica clásica, los interrogatorios verbales de un sujeto, y los pensamientos en voz alta, y consideraba que la veridicidad del último tipo de reports verbales era muy diferente de los dos 
primeros. De hecho, consideraba que el pensamiento consistía primariamente en un habla subvocal.

En su famoso artículo de 1913, Watson afirmaba con énfasis que

«La psicología tal como la ve el conductista es una rama experimental puramente objetiva de la ciencia natural. Su fin teórico es la predicción y control de la conducta. La introspección no forma parte esencial de sus métodos, ni el valor científico de sus datos depende de la facilidad con la que ellos mismos pueden llegar a la interpretación en términos de la conciencia» (Watson, 1913, p. 158).

Inicialmente Watson no afirmaba que la conciencia no existía ni que la introspección era un mito (Lyons, 1983), sino que meramente afirmaba que no formaba parte de una genuina psicología científica; para él la psicología había utilizado la introspección tanto como la física y la química. Sin embargo, siete años después de 1913 estaba mucho más firme en el rechazo de la conciencia, y ello podría tomarse como un indicio de que también el concepto de introspección se hallaba en un proceso de cambio a través de diversas matizaciones, tal como Boring (1953) y Lyons (1983) han puesto en evidencia. En efecto, en 1910, Titchener afirmaba que la introspección era «la suma total de la experiencia humana considerada como dependiente de la persona que experiencia» (haciendo un juego de palabras), pudiendo escribir la fórmula:

\section{Introspection $=$ psychological \\ (clear experience $\rightarrow$ report)}

lo cual significa que la introspección era la tenencia de una experiencia clara bajo el punto de vista psicológico, y el informarlo como tal bajo el mismo punto de vista. Watson desechó formalmente la introspección de la psicología, pero se quedó en los resultados más fiables de tal introspección, especialmente en psicofísica (Watson, 1919), por lo que le era necesario separarse de la introspección como verbal report, y nos podríamos preguntar con Boring (1953), ¿̨es la introspección algo más que el informe verbal?, a la vez que nos reiteramos en la convergencia entre aspectos epistemológicos y recursos metodológicos.

Posteriormente sí ha existido diferencia entre ambos. El informe verbal visto simplemente como conducta es capaz de una especificación física, en la cual la escritura y denominación de palabras aparece como tipos de movimientos muy distintos hasta que se han mostrado equivalentes en una situación experimental. Por otra parte, el informe verbal como introspección no es respuesta, sino observación indirecta, y por lo tanto descripción y referencia (indicación) de los objetos de observación en el sentido del significado de los términos utilizados.

Otra forma de expresarlo sería mediante las siguientes fórmulas:

a) Observación introspectiva:

$\mathrm{E} \rightarrow \mathrm{O}=\mathrm{S} \rightarrow$ hechos de conciencia.

Es decir, el experimentador anota los hechos de conciencia que el observador, que es el sujeto, ha observado.

b) Observación conductista:

$\mathrm{O}=\mathrm{E} \rightarrow \mathrm{S} \rightarrow$ hechos de la psicología. 
El observador, que es el experimentador, observa la conducta del sujeto con respecto a sus implicaciones para los hechos de la psicología.

En la introspección clásica el sujeto es el observador que asume la responsabilidad de que sean correctas sus descripciones de los datos conscientes, siendo mejor el estar adiestrado que el tener experiencia. El conductismo cambia el locus de responsabilidad científica del sujeto que observa al experimentador, que se convierte en observador del sujeto; de esta forma es posible unir la observación psicológica irresponsable y sujetos no adiestrados (animales, niños, enfermos mentales, etc., así como adultos normales). Por esto los tests mentales tuvieron fácil entrada en la psicología, porque en su gran parte implicaban respuestas verbales para sujetos naive, y se llevaban a cabo experimentos con animales (que perduran) porque ordinariamente su conducta discriminativa es un lenguaje ideado por el experimentador y enseña al animal de forma que el experimentador pueda preguntarse por sus aptitudes y capacidades. ¿Podríamos afirmar que el animal no introspecciona porque no se comunica consigo mismo lo que está comunicando al experimentador? Lo importante es ver que Watson, al atacar la introspección, estaba objetando, no el uso de palabras por el sujeto, sino el esperar que éste utilice las palabras solamente con aquellos significados de los términos que el experimentador desea.

Watson, en la sustitución del informe verbal por la introspección se movía en dirección positivista, pero la culminación de este movimiento vino con la aceptación de las definiciones operacionales, que prestarían una especificación más segura para los conceptos psicológicos. El efecto prolongado de discusiones llevó a un cambio (en 1930) en el estatus de la conciencia desde un almacén de experiencia más allá de la cual se perfila la ciencia empírica, hasta llegar a un concepto basado en la observación y especificado por operaciones observacionales que hacen que los datos conscientes sean válidos para la ciencia (Boring, 1953). Este es el gran cambio sobre la introspección que no puede mentir, porque el tener experiencia es el conocimiento de que se tiene.

El objeto de la introspección es la conciencia (consciousness) según Boring (1953), y en la actualidad (psicología cognitiva) persiste (Hilgard, 1980) en los informes de experiencia sensorial en psicofísica, en los protocolos de pacientes con dificultades psicológicas, en las descripciones de ciertos eventos, etc. Luego se implica una repercusión a nivel metodológico, no exenta de posibles valorciones contrapuestas, que obligan a una postura cauta y recelosa ya que si las críticas clásicas a la introspección están asumidas, el problema común es la creencia de que la experiencia consciente no proporciona «datos» fiables sobre los fenómenos mentales (Paivio, 1971; Natsoulas, 1967, 1970; Neisser, 1979; Dennett, 1979; Wason y Evans, 1975; Evans y Wason, 1976; Nisbett y Wilson, 1977), creyéndose que existe una dualidad producto-proceso, de modo que los contenidos de la conciencia son "productos» del pensamiento, pero los "procesos» subyacentes resultarán inaccesibles y por tanto no susceptibles de investigación introspectiva (Rodrigo, 1982).

Es preciso, pues, depurar las técnicas introspectivas y estudiar la influencia de diversos tipos de instrucciones verbales, pautas empíricas de respuesta y su correspondencia con los procesos mentales.

Por fin, y por lo que se refiere a las repercusiones metodológicas en el 
aprendizaje verbal, el conductismo que se desarrolló a partir de la experimentación animal y que generalizó los principios explicativos del comportamiento animal al comportamiento humano se vio obligado a demostrar su validez a partir del trabajo experimental desarrollado por Ebbinghaus, quedando reducido a un problema de adquisición de «sílabas sin sentido", partiendo del concepto de asociación como principio básico del aprendizaje y del de transferencia de entrenamiento para explicar tanto el aprendizaje como el olvido (Garzón y Seoane, 1982). De nuevo convergen los aspectos epistemológicos y los procedimentales...

De acuerdo con el planteamiento de Melton e Irwin (1940) a partir de la teoría de los dos factores, por la que se entiende que cuando un sujeto aprende dos respuestas para un mismo estímulo se desencadena un factor de competición de respuestas que conlleva el debilitamiento (factor de desaprendizaje asociativo) de la fuerza asociativa de una de las respuestas, de forma tal que éste desaparece con el paso del tiempo y la asociación debilitada recupera su fuerza (fenómeno de recuperación espontánea), se llegó a que los teóricos de la interferencia, después de verificar tales factores, llegaron a una renuncia progresiva de los principios básicos del asociacionismo, así como a un caos empírico de hipótesis y contrahipótesis (Garzón y Seoane, 1982), renunciando a la posibilidad de aplicar los principios asociacionistas a la conducta verbal, y pasaron de entender el aprendizaje y el olvido como un fenómeno de dependencias asociativas específicas (énfasis en la asociación) a entenderlos como un problema de adquisición y diferenciación de conjuntos de respuestas (énfasis en el papel del término «respuesta»), donde ya no cuentan las asociaciones específicas (Postman y Stark; 1969) y se acabaron por plantear el aprendizaje y el olvido como un problema de la codificación del estímulo (énfasis en el papel del término «estímulo") (Garzón y Seoane, 1982).

De aquí que Martín $(1965,1968,1972,1973)$ signifique la ruptura o crisis del paradigma conductista del aprendizaje verbal y la aparición de nuevos planteamientos que rompen con los postulados teóricos de la psicología de E-R. Hasta ahora los contenidos de las investigaciones realizadas en este ámbito venían determinados, en parte, por la metodología utilizada, con lo que los resultados estaban restringidos a ella; así, por ejemplo, debido al papel pasivo del sujeto, lo eliminaron como variable (eliminación de las diferencias individuales) porque no les interesaba (Cronbach, 1957, 1975). Los resultados obtenidos son los que deseaban, aunque existen unas falacias implícitas en su sustrato que tendrian forzosamente que originar fluctuaciones al variar el marco conceptual subyacente a tal metodología.

La llegada de la psicología cognitiva al ámbito del aprendizaje - aun eliminado el término- supuso una apertura de las situaciones utilizadas para su estudio (Klatzky, 1980; Bransford, 1979; Anderson, 1980), y así tenemos estudios referidos al aprendizaje de aritmética elemental (Resnick y Ford, 1981), de construcción de pruebas geométricas (Greeno, 1978), de comprensión de historias (Stein y Trabasso, 1982) y de predicción de lluvia (Stevens y Collins, 1982). Se muestra básicamente como interesan las diferencias de la behavior-in-situation (conducta según la situación) que identifica el nuevo aprendizaje, y se caracteriza mediante el término «tarea de aprendizaje», implicándose que su especificación incluye tanto la situación externa como la conducta que interactúa con ella (Hulse, Deese y Egeth, 
1985; Hill, 1981). Cuando Melton (1964) actuó de compilador en la obra Categories of Human Learning, se ocupó de situaciones familiares, como las de condicionamiento operante en palomas, aprendizaje de rutinas verbales, aprendizaje incidental de pares de palabras, y aprendizaje de habilidades perceptivo-motoras. Incluso cuando las teorías del aprendizaje se presentaban directamente, como en el caso de Bower y Hilgard (1981), encontramos ideas teóricas vinculadas a las situaciones, como perros salivando a la vista de comida, palomas picoteando en manchas circulares, ratas recorriendo compartimientos, o sujetos humanos aprendiendo pares asociados.

Había surgido la necesidad de lograr una mayor validez, por lo que la gran diversidad de tales situaciones era un cambio esperado y algunos autores (Gagné, 1984) se preguntan si el estudio del aprendizaje debería continuar en esta situación, donde existe una gran especificidad en la interacción entre el que aprende y el entorno, es décir, en la tarea. Gagné (1984) afirma que todos aprendemos, puesto que todos realizamos actividades manifiestas cada día y como observadores de la conducta sabemos que se ha aprendido por la percepción que llevamos a cabo, es decir, conocemos que ha habido aprendizaje cuando observamos sus resultados o efectos.

Ya, finalmente, y en el ámbito del aprendizaje animal, si nos hallamos en la línea de aumento de la validez externa de los resultados obtenidos, por una parte puede lograrse evitando la artificialidad de las situaciones experimentales, con lo que los estudios de campo se presentarían como alternativa y ya Altmann (1974), en este sentido, ha discutido las ventajas e inconvenientes de ambos $y$, por otra parte, se conseguiría preparando situaciones experimentales relativamente complejas que traten de reproducir algunos aspectos generales del medio natural, así como comprobando explícitamente la viabilidad de la aplicación de los principios obtenidos en experimentos bien controlados al análisis de comportamientos más naturales (Aguado, 1983). En cualquiera de estos casos, las diversas estrategias metodológicas posibles hacen patente la posibilidad de aumentar la validez externa de los resultados de los estudios de laboratorio, aún conservando un grado considerable de precisión y control de la situación experimental, a la vez que ponen de manifiesto que la investigación de posibles procesos generales en condiciones de alto control experimental no es una tarea inútil o sin sentido, siempre que este enfoque se complemente con el análisis explícito de la viabilidad de la aplicación de los principios descubiertos a situaciones caracterizadas por una mayor validez externa (Aguado, 1983).

\section{BREVE COMENTARIO, A MODO DE ILUSTRACION, SOBRE LA INCIDENCIA DE LA CONFRONTACION EN UN CONTEXTO CLINICO}

A pesar de la dificultad que surge habitualmente cuando se desea llegar a un acuerdo sobre definiciones de modificación de conducta (Cruz, 1984), sí es un hecho totalmente aceptado que las técnicas de modificación y terapia de conducta surgieron de las teorías del aprendizaje como una alternativa a las interpretaciones y tratamientos tradicionales de la conducta 
anormal, aunque posteriormente se hayan desarrollado y diversificado enormemente. Pero no es tan simple el proceso.

En la expansión de la década de los setenta, la terapia de conducta se convierte en una tecnología aplicada que a nivel teórico estaba basada en los principios del método científico y no exclusivamente en los principios de la teoría del aprendizaje (Yates, 1970), con lo que trata de enfatizarse su preocupación por garantizar el objetivismo y rigurosidad propias de la época conductista. En este sentido,

«la terapia y/o modificación de conducta podría definirse en la actualidad como la psicología experimental humana orientada hacia modelos explicativos de aprendizaje social" (Pelechano, 1979, p. 4).

y, por su parte, en Mayor y Labrador (1984) se afirma que la modificación de conducta es una orientación terapéutica que considera a la conducta normal y a la anormal regidas por los mismos principios, que recurre a la evaluación objetiva y a la verificación empírica, por lo cual utiliza procedimientos y técnicas basadas en la psicología experimental para eliminar conductas desadaptadas, sustituyéndolas por otras y enseñando conductas adaptadas cuando éstas no se han producido.

No podemos olvidar, además, que la terapia de conducta, por su propia naturaleza, y de acuerdo igualmente con el método científico que le sirve de base, constituye (Haynes, 1978) un sistema de procedimientos y construcciones en continua evolución y sometido a una constante autoevaluación y autocorrección. Se hallan, pues, interactuando, de forma que la terapia de conducta constituye un sistema cibernético en continuo progreso que somete ininterrumpidamente sus principios y métodos a una evaluación y corrección constante en función del empirismo que la caracteriza y le sirve de base (Fernández Ballesteros y Carrobles, 1981). Y es esta dependencia de la investigación empírica la que explica, precisamente, la importancia fundamental que para el terapeuta de conducta tiene la evaluación, que está siempre presente, de una forma u otra, en todo el proceso de aplicación de la modificación de conducta y con la meta última de la explicación de la conducta y su cambio (Fernández Ballesteros, 1984), lo cual resulta ya una superación del análisis procedente del conductista radical. Asimismo, y desde el ámbito de la Medicina Comportamental, actualmente la mayoría de los procedimientos son los mismos que se utilizan en terapia de conducta para tratar otros tipos de problemas (Saldaña, 1984).

El propio Kazdin (1983) sostiene la existencia de un gran paralelismo entre la modificación de conducta y la teoría del aprendizaje, de modo que la primera resultaba sinónimo de la aplicación de los principios experimentales del aprendizaje al cambio de conducta, lo cual permitió contraponer el modelo terapéutico psicoanalítico o intrapsíquico y el de la modificación de conducta, que postulaba una continuidad entre la conducta normal y la anormal, explicada por los mismos principios, y un cambio o modificación de toda conducta según los principios del aprendizaje.

Ahora bien, dado que la metodología y principios explicativos de la conducta son diferentes según se trate del modelo de condicionamiento clásico o de condicionamiento operante, derivando de ellos dos enfoques terapéuticos con objetivo y técnicas modificadoras diferentes - el enfoque de Wol- 
pe $(1958,1969)$, Wolpe y Lazarus (1966), Eysenck $(1959,1960)$ y Yates ()1976), ordinariamente denominado «terapia de conducta» y la orientación skinneriana de la modificación operante de la conducta, comúnmente denominada «análisis de la conducta» (Bayés, 1983)-, de la misma forma, actualmente, la emergencia de la psicología cognitiva ha incidido ayudada por la vulnerabilidad de un punto débil - no sé si el principal-y es el grado de fundamentación de los principios de modificación de conducta en la teoría del aprendizaje (Rodríguez Domínguez, 1984), puesto que las técnicas utilizadas en el cambio de conducta no se consideran productos exclusivos de los principios del condicionamiento clásico y operante, sino que además se apoyan en datos investigados en procesos fisiológicos, cognoscitivos y simbólicos (Bandura, 1969, 1974).

La tendencia contemporánea imperante respecto a la modificación de conducta resalta su no identificación con una teoría específica (Rodríguez Domínguez, 1984), pudiendo utilizar principios procedentes de muchas áreas de estudio, de donde extraería un cuerpo siempre cambiante de descubrimientos experimentales. Según ello, la modificación de conducta vendría prioritariamente definida por la referencia a la metodología que los diferentes modelos teóricos y tecnologías aplican al cambio de conducta, representando un enfoque científico homogéneo del estudio de la conducta y su modificación (Mahoney, Kazdin y Leswing, 1984).

Ello no obsta para que puedan diferenciarse plenamente los modelos psicoterapéuticos conductual y cognitivo. Y si hasta ahora no hemos referido al primero, la terapia cognitiva se apoya en unas bases epistemológicas de actividad o constructividad por parte del sujeto, que deberá llevar a cabo una cierta reordenación o reestructuración, por lo que los objetivos y procedimientos de intervención deberán ser lógicamente distintos. Los objetivos de cambio terapéutico cognitivo se centran en percepciones, pensamientos, creencias, expectativas, atribuciones y otras actividades cognitivas, es decir, acontecimientos privados e internos, con lo que las técnicas terapéuticas de intervención se dirigen a modificar procesos cognitivos que se supone son responsables de una conducta o un conjunto de conductas manifiestas específicas (Beck, 1970), sea a través de propiciar un cambio en el conjunto de creencias irracionales del sujeto (Ellis, 1962), o requiriendo una variación en el estilo de pensamiento del individuo (Beck, 1976), o al perseguir una remodelación de la estructura cognitiva y metacogniciones del sujeto (Meichenbaum, 1975). Con ello, las posibilidades de intervención terapéutica son amplias, desde las situaciones en contextos clínicos a las intervenciones en situaciones múltiples de la vida cotidiana relacionadas con deficiencias conductuales por ansiedad (exámenes, hablar en público, relaciones interpersonales, etc.).

En los últimos años, sin embargo, es conocido por todos el debate, de matiz principalmente epistemológico (Rodríguez Domínguez, 1984), sobre la consideración de la terapia cognitiva como alternativa a la conductual o como una fase pasajera de la modificación de conducta (Wilson, 1978); debate que, por otra parte, ha involucrado a Skinner (1977) y Wolpe (1978), asegurando que la terapia de conducta nunca prescindió de variables cognitivas, aunque tales variables serían explicables bajo los principios del condicionamiento, al igual que las técnicas cognitivo-semánticas serían combinables con las de la terapia de conducta y con el manejo de contingencias 
de refuerzo y la superior eficacia en los casos clínicos de esa conjunción de técnicas es confirmada por Mahoney y Kazdin (1979).

Si como consecuencia de ello la modificación de conducta congnitiva constituye la orientación psicoterapéutica más ampliamente aceptada y defendida a lo largo de la última década como intento de síntesis entre terapias conductual y cognitiva (Kendal y Hollon, 1979; Meichenbaum, 1977; Meichenbaum y Cameron, 1980), su surgimiento cabe atribuirlo al deseo de integrar estrategias de tratamiento cognitivas con el manejo de contingencias conductuales en orden a superar ciertos problemas de conducta y obtener resultados efectivos (Rodríguez Domínguez, 1984). Es cierto que el planteamiento inicial es opuesto, ya que mientras que los teóricos cognitivistas consideran que el aprendizaje implica fundamentalmente la reestructuración activa del campo cognitivo y la percepción de relaciones entre acontecimientos ambientales, los teóricos E- $R$ se apoyan en conexiones y asociaciones para la explicación del aprendizaje; pero es igualmente cierto que en la práctica nunca se ha dado tal rigidez extrema, ya que el propio Skinner (1963) reconoce la incidencia de percepciones y acontecimientos privados en el aprendizaje; Wolpe (1978) integró fenómenos cognitivos relacionados con la imaginacón y las autoverbalizaciones encubiertas en las técnicas de desensibilización sistemática, y Bandura (1969) ha resaltado el papel de los procesos cognitivos en el aprendizaje social.

Pero si, a pesar de todo, la terapia de conducta - clásica u operanterepresenta un extremo de las orientaciones teóricas que sigue siendo reticente a la utilización de conceptos mentales y variables mediacionales en la explicación de la conducta y en el diseño de técnicas para modificarla (con lo cual su principal incidencia se llevará a cabo sobre la conducta manifiesta y la influencia del entorno sobre ella) (Rodríguez Domínguez, 1984), y si, por el extremo, la psicoterapia cognitiva se caracteriza por el énfasis dado a tales procesos (de forma que interesa el estudio de conductas encubiertas), y en la práctica se nos proponen terapias integradas conductual-cognitivas, el «milagro» sólo sería posible si se conjuntan una diversidad de técnicas procedentes de puntos de partida muy distintos (Bas Ramallo, 1981), y si hasta ahora no se ha logrado el acuerdo entre terapeutas de ambas vertientes quizá pueda atribuirse precisamente a la necesaria maduración que implica este proceso de convergencia metodológico, que es, al fin y a la postre, el más inmediatamente percibido, ya que marca los procedimientos diarios de trabajo.

Conscientes del necesario pluralismo metodológico, que ha sido el hilo conductor de esta charla - precisamente por su defecto, que tan malas consecuencias conlleva-, y su convergencia con aspectos epistematológicos, se evidencia además la importancia del nivel técnico-aplicado, ya que puede ocurrir y ocurre que la propia investigación tecnológica «reconduzca» y haga corregir o ajustar sus planteamientos al presentarse problemas que le afecten en cuanto investigación científica sobre un dominio particular (Caparrós, 1984), y en el caso del aprendizaje es un hecho indudable.

Llegados a este punto, enfatizamos de nuevo, como en el inicio, la necesidad de profundizar en su estudio por permitir con cierta flexibilidad un escoramiento hacia diversas direcciones, a pesar de posicionamientos teóricos antagónicos, y con la seguridad de que, a la corta o a la larga, existirá un respaldo metodológico que es el que posibilitará la superación de viejas 
barreras, existiendo no sólo voluntad de acercamiento sino una viabilidad a nivel fáctico, es decir, técnico.

\section{Referencias}

Aguado, L. (1983). Tendencias actuales en la psicologia del aprendizaje animal. Anuario de Psicología, 29, 67-88.

Aiken, H.; Babage, CH.; von Neumann, J.; Shannon, C. E.; Turing, A. M.; Walter, W. G., et al. (1970). Perspectivas de la revolución de los computadores. Madrid: Alianza Universidad, 1975.

Altmann, J. (1974). Observational study of behavior: Sampling methods. Bebaviour, 49, 227-262.

Alvira, F.; Avia, M. D.; Calvo, R., y Morales, F. (1979). Los dos métodos de las Ciencias Sociales. Madrid: Centro de Investigaciones Sociológicas.

ANDERSON. J. R. (1972). FRAN: A simulation model of free recall. En G. H. Bower (comp.) The psychology of learning and motivation, vol. 5. Nueva York: Academic Press.

ANDERSON, J. R. (1980). Cognitivo psychology and its implications. San Francisco: Freeman.

ANGUERA, M. T. (1983a). La experimentación psicológica a la luz del cambio paradigmático. Comunicación presentada en el II Congreso de Teoría y Metodología de las Ciencias. Oviedo, abril, 1983.

ANGUERA, M. T. (1983b). Why did Experimental Psychology abandon behaviourism? Critical thoughts on the methodological rigidity.Comunicacion presentada en el Fist European Meeting on the Experimental Analysis of Behaviour (EMEAB). Liège, Belgium, julio, 1983.

Anguera, M. T. (1984). Cambio metodológico y cambio teórico desde la Psicología Experimental. ¿Puede cambiar una teoría sin cambiar el método? En M. T. Anguera y J. J. Veá (comps.) Conducta animal y prepresentaciones mentales. Barcelona: P. P. U., 35-57.

ArNau, J. (1979). Problemática metodológica de la validez experimental. Anuario de Psicologia, 21, (2), 51-82.

Arnau, J. (1982). La explicación en Psicología Experimental: Del conductismo al cognitivismo. En I. Delclaux y J. Seoane (comps:). Psicología cognitiva y procesamiento de la información. Madrid: Pirámide, 93-106.

ATKINSON, R. C. (1977). Reflections on psychology's past and concerns about its future. American Psychologist, 32, (3), 205-210.

ATO, M. (1984). El tiempo de reacción como variable dependiente: Algunas cuestiones de procedimiento experimental. Anales de la Universidad de Murcia, 1, 209-224.

Bandura, A. (1969). Principles of bebavior modification. Nueva York: Holt, Rinehart y Winston.

BANDURA, A. (1974). Behavior theory and the models of man. American Psychologist, 29, 859-869.

Barber, T. X. (1976). Pitfalls in buman research. Ten pivotal points. Nueva York: Pergamon Press.

Bas Ramallo, F. (1981). Las terapias cognitivo-conductuales: Una revisión. Estudios de Psicologia, 7, 92-114.

BAYES, R. (1983). Enseñanza y ejercicio profesional del Análisis de la Conducta en España. Revista de Psicología General y Aplicada, 38, (2), 322-245.

BAYLOR, G. W.; GASCON, J., y LEMOYNE, G. (1973). An information processing model of some seriation task. The Canadian Psychologist, 14, (2), 167-196.

BECK, A. T. (1976). Cognitive therapy and emotional disorders. Nueva York: International University Press.

BECK, A. T. (1970). Cognitive therapy: Nature and relation to behavior therapy. Behavior Therapy, $1,184-200$.

BINDRA, D. (1974). A motivational view of learning, performance and behavior modification. Psychological Review, 81, 199-213.

BINDRA, D. (1978). How adaptative behavior is produced: A perceptual-motivational alternative to response-reinforcement. The Bebavioral and Brain Sciences, 1, 41-91 [incluye comentarios].

Bolles, R. C. (1972). Reinforcement, expectancy and learning. Psychological Review, 79, 394-409.

BONEAU, C. A. (1984). Paradigm regained? Cognitive behaviorism restated. American Psychologist, 29, 297-309.

Boring, E. G. (1953). A history of introspection. Psychological Bulletin, 50, (3), 196-189.

BOWER, G. M. y HILGARD, E. J. (1981). Theories of learning (Stb edition). Englewood Cliffs, N. J.: Prentice-Hall.

BRANSFORD, J. D. (1979). Human cognition. Belmont, Ca.: Wadsworth. 
Breger, L. (1969). The ideology of behaviorism. En L. Breger (comp.). Clinical-cognitive psychology. Englewood Cliffs, N. J.: Prentice-Hall, 25-55.

BRISKMAN, L. B. (1972). Is a kuhnian analysis applicable to psychology? Science Studies, 2, 87-97.

BUGELSKY, B. R. (1980). Aprendizaje humano. En B. B. Wolman (comp.). Manual de Psicologia General. Vol. III: Aprendizaje, lenguaje, pensamiento e inteligencia. Barcelona: Martínez Roca, 162-194.

BUGELSKY, B. R. y SHARLOCK, D. P. (1952). An experimental demonstration of unconscious mediates association. Jourmal of Experimental Psychology, 44, 334-338.

CAPARROS, A. (1979). Introducción histórica a la psicología contemporánea. Barcelona: Rol.

CAPARRos, A (1984). La Psicología y sus perfiles. Instroducción a la cultura psicológica. Barcelona: Barcanova.

CAPARROS, A. y GABUCIO, F. (1984). El surgimiento del paradigma cognitivo: Una cuestión problemática. Comunicación presentada al Symposium sobre Actividad Humana y Procesos Cognitivos. Madrid, diciembre, 1984.

Catania, A. Ch. (1984). Análisis conductual en la extrapolación de la conducta animal a la humana. En M. T. Anguera y J. J. Veá (comps.). Conducta animal y representaciones mentales. Barcelona: P. P. U., 13-32 (orig. publicado en 1983).

CERCONE, N. y McCALLA, G. (1984). Artificial intelligence: Underlyings assumptions and basic objectives. Journal of the American Society for Information Science, 35, (5), 280-290.

CHOMsky, N. (1957). Syntactic structures. The Hague: Mouton.

CHOMSKY, N. Review of Skinner "Verbal Behavior". Language, 35, 26-58.

CROBNACH, L. L. (1957). The two disciplines of scientific psychology. American Psychologist, 12, 671-685.

CROBNACH, L. L. (1975). Beyond the two disciplines of scientific psychology. American Psychologist, 30, 116-127.

Cruz Hernandez, M. (1982). Problemas epistemológicos del concepto y definición de la psicología. Estudios de Psicologia, 12, 96-105.

Cruz, J. (1984). Breve historia de la Modificación y Terapia de Conducta en España. Anuario de Psicologia, 30/31, (1-2), 7-29).

DAY, W. (1977). On the difference between radical and methodological behaviorism. Comunicación presentada en el Annual Meeting of the Midwestern Association of Behavior Analysis. Chicago, septiembre, 1977.

DelClaUX, I. (1982). Introducción al procesamiento de la información en psicología. En I. Delclaux y J. Seoane (comps.): Psicología cognitiva y procesamiento de la información. Madrid: Pirámide, 21-38.

DE MEY, M. (1982). The cognitive paradigm. Dordrecht: Reidel Publishing Company.

DENNETT, D. C. (1979). Artificial intelligence as philosophy and as psychology. En M. Ringle (comp.) Philosophical perspectives in artificial intelligence. Brighton, Sussex: Harvester Press, 57-78.

DE VEGA, M. (1982). La metáfora del ordenador: Implicaciones y límites. En I. Delclaux y J. Seoane (comps.) Psicología cognitiva y procesamiento de la información. Madrid: Pirámide, 63-84.

DE VEGA, M. (1984). Introducción a la psicología cognitiva. Madrid: Alianza Editorial.

DiCKINSON, A. (1980). Contemporary animal learning theory. Cambridge: Cambridge University Press.

Diez-Chamizo, V.; Blackman, D. E., y Thomas, G. V. (1981). Effects of the conditioned suppression procedure on an operant discrimination. Behaviour Analysis Letters, 1, 267-274.

Difz-Chamizo, V.; Sterio, D. y Mackintosh, N. J. (en prensa). Blocking and overshadowing between intra-maze and extra-maze cues: $A$ test of the independence of locale and guidance learning. The Quarterly Journal of Experimental Psychology (Section B, Comparative and Physiological Psychology).

DONDERS, F. (1868-1869). Over de shelheid van psychische processes. Tweede Reeks, 2, 92-120. [Trad, inglesa, On the speed of mental processes. Acta Psychologica, 1969, 30, 412-431].

DowNS, R. M. (1981). Maps and mapping as metaphors for spatial representation. En L. S. Liben; A. H. Patterson, y N. Newcombe (comps.) Spatial representation and behavior across the life span. Theory and application. Nueva York: Academic Press, 143-166.

Downs, R. M., y STEA, D. (1977). Maps in minds. Reflections on cognitive mapping. Nueva York: Harper y Row.

Ellis, A. (1962). Razón y emoción en psicoterapia. Bilbao: Desclee de Brouwer, 1980.

ESTES, W. K.; HOPKINS, B. L. y CROTHERS, E. J. (1960). All-ornone and conservation effects in the learning and retention of paired-associates. Journal of Experimental Psychology, 52, 329-339.

Evans, J. S. B., y Wason, P. C. (1976). Rationalization in a reasoning task. British Journal of Psychology, 67, (4), 479-486.

EYSENCK, H. J. (1959). Learning theory and behavior therapy. Journal of Mental Science, 10S, 61-75. 
Eysenck, H. J. (1960). Behavior therapy and the neuroses. Nueva York: Pergamon Press.

EYSENCK, M. W. (1984). A handbook of cognitive psychology. Hillsdale, N. J.: Lawrence Erlbaum Associates.

FERNÁNDEZ BALlesteros, R., y CARRobles, J. A. I. (1981). Evaluación versus tratamiento. En R. Fernández Ballesteros y J. A. I. Carrobles (comps.): Evaluación conductual. Metodología y aplicaciones. Madrid: Pirámide, 127-154.

FERNÁNDEZ BALLESTEROS, R. (1984). Aportaciones de la evaluación conductual a la reformulación del concepto de diagnóstico psicológico. Anuario de Psicologia, 30/31, (1-2), 31-44.

Fierro, A. (1982). La explicación en psicología. Estudios de Psicología, 12, 107-126.

FODOR, J. A. (1981). El problema cuerpo-mente. ¿Podrían sufrir las máquinas de calcular, tener esperanzas los marcianos y pensar los espíritus incorpóreos? El enfoque funcionalista de la psicología contemporánea suscita e incrementa la posibilidad lógica de que sí podrían. Investigación y Ciencia, 54, 62-75.

FORRESTER, W. E. (1984). Publication trends in human learning and memory: 1962-1982. Bulletin of the Psychonomic Society, 22, (2), 92-94.

GABUCIO, F. (1983). El concepto de paradigma en Psicología: Alcance y limitaciones. Memoria de Licenciatura inédita. Universidad de Barcelona.

GADLIN, H., y INGLE, G. (1975). Through the one-way mirror. The limits of experimental self-reflections. American Psychologist, 30, 1003-1009.

GAGNE, R. M. (1984). Learning outcomes and their effects. Useful categories of human performance. American Psychologist, 39, (4), 377-385.

Garzón, A., y SEOANE, J. (1982). Nueva lectura de los datos empíricos en el aprendizaje verbal. En J. Seoane (comp.) Teoría y métodos en psicología experimental. Valencia: Alfaplus, 23-35.

GERGEN, K. J., y GERGEN, M. M. (1982). Explaining human conduct: Form and function. En P. F. Secord (comp.). Explaining buman behavior. Consciousness, buman action and social structure. Beverly Hills, Ca.: Sage, 127-154.

GREENO, J. G. (1978). A study of problem solving. En R. Glaser (Ed.) Advances instructional psychology, vol. 1. Hillsdale, N. J.: Lawrence Erlbaum Associates, 13-75.

HARRE, R., y SECORD, P. F. (1972). The explanation of social bebaviour. Oxford: Blackwell.

HAYNES, S. N. (1978). Principles of bebavioral assessment. Nueva York: Gardner Press.

HESS, E. H. (1959). Imprinting. Science, 30, 133-141.

Hilgard, E. R. (1980). Consciousness in contemporary psychology. Annual Review of Psychology, 31, 1-26.

HILGARD, E. R., y ATKINSON, R. C. (1967). Introduction to psychology. Nueva York: Harcourt, Brace y World.

HILL, W. F. (1981). Principales of learning: A handbook of applications. Palo Alto, Ca.: Mayfield.

HousTON, J. P. (1981). Fundamentals of learning and memory. Nueva York: Academic Press.

HuLL, C. L. (1943). Principles of behavior. Nueva York: Appleton-Century-Crofts.

Huld, C. L. (1951). Essentials of behavior. Nueva Haven: Yale University Press.

HULSE, S. N.; DEESE, J., y EGETH, H. (1975). The psychology of learning (Sth edition). Nueva York: McGraw-Hill.

IsAaCSON, R. L.; HUTT, M. L., y BLUM, M. L. (1965). Psychology: The science of behavior. Nueva York: Harper y Row.

KazDiN, A. E. (1983). Historia de la modificación de conducta. Bilbao: Desclée de Brouwer (edic. origin., 1978).

Kendall, T., y Hollon, S., (Comps.) (1979). Cognitive-behavioral interventions: Theory, research and procedures. Nueva York: Academic Press.

KendLER, H. H. (1968). Basic psychology. Nueva York: Appleton-Century-Crofts.

KENDLER, H. H., y KENDLER, T. S. (1962). Vertical and horizontal processes in problem solving. Psychological Review, 69, 1-16.

KLAHR, D. Y WALLACE, J. G. (1976). Cognitive developments, an information-processing view. Hillsdale, N. J.: Lawrence Erlbaum Associates.

KLATZKY, R. L. (1980). Human memory: Structures and processes (2nd edition). San Francisco: Freeman.

Kosslyn, S. M., y SChwartz, S. P. (1977). A simulation of visual imaginery. Cognitive ScienCe, 1, 265-295.

KRUGLANSKI, A. W. (1976). On the paradigmatic objections to experimental psychology. American Psychologist, 31, 655-663.

KuHn, T. S. (1979). La estructura de las revoluciones cientificas. México: Fondo de Cultura Económica (edc. origin., 1962).

KENDLER, H. H. (1981). Psychology: A science in conflict. York: Oxford University Press.

LACHMAN, R.; LACHMAN, J. L., y BUTTERFIELD, E. C. (1979). Cognitive psychology and information processing: An introduction. Hillsdale, N. J.: Lawrence Erlbaum Associates.

LYONS, W. (1983). The transformation of introspection. British Journal of Social Psychology, 22, 327-342.

MacCORQUEDALE, K., Y MeEHL, P. E. (1948). On a distintion between hypothetical constructs and intervening variables. Psychological Review, S5, 95-107. 
MACKENZIE, B. D. (1982). El behaviorismo y los limites del método científico. Bilbao: Desclée de Brouwer (edic. origin. 1977).

MAHONEY, M. J.; KAZDIN, A. E., y Lesswing, W. J. (1974). Behavior modification: Delusion or deliverance? En C. M. Franks y G. T. Wilson (Comps.). Annual Review of Bebavior Therapy: Theory and Practice, vol. 2. Nueva York: Bruner-Mazel, 11-40.

MAHONEY, M. J., y KAZDIN, A. (1979). Cognitive behavior modification: Misconceptions and premature evaluation. Psychological Bulletin, 86, 1044-1049.

Martin, E. (1965). Transfer of verbal paired-associates. Psychological Review, 72, 327-343.

MARTIN, E. (1968). Stimulus meaningfulness and paired-associate transfer: An encoding variability hypothesis. Psychological Review, 75, 421-441.

MARTIN, E. (1972). Stimulus encoding in learning and transfer. En A. W. Melton y E. Martin (Comps.). Coding processes in buman memory. Washington: Wiley y Sons.

MARTIN, E. (1973). Memory codes and negative transfer. Memory y Cognition, 4, 495-498.

MASSARO, D. W. (1975). Experimental psychology and information processing. Chicago: Rand McNally.

MAYOR, J. (1980). Orientaciones de la Psicología Cognitiva. Análisis y Modificación de Conducta, 6, 213-278.

MAYOR, J. (Comp.) (1984a). Actividad bumana y procesos cognitivos. Madrid: Alhambra.

MAYOR, J. (1984b). Actividad humana y procesos cognitivos. En J. Mayor (Comp.) Actividad bumana y procesos cognitivos. Madrid: Alhambra, 3-36.

MAYOR, J. y LABRADOR, F. J. (Comps.) (1984). Manual de modificación de conducta. Madrid: Alhambra.

MeICHENBAUM, D. (1975). Self-instructional methods. En F. H. Kanfer y A. P. Goldstein (Comps.): Helping people change: A textbook of methods. Nueva York: Pergamon.

MeICHENBAUM, D. (1977). Cognitive behavior modification: An integrative approach. Nueva York: Plenum Press.

Meichenbaum, D., y Cameron, R. (1980). Cognitive behavior modification. En C. Franks y T. Wilson (comps.): Handbook of behavior therapy. Nueva York: Guilford Press.

Melton, A. W. (comp.) (1964). Categories of human learning. Nueva York: Academic Press.

MilleR, G. A.; GALANTER, E., y PRIBRAM, K. H. (1960). Plans and the structure of behavior. Nueva York: Holt, Rinehart \& Winston.

MOROZ, M. (1972). The concept of cognition in contemporary psychologoy. En J. R. Royce $y$ W. W. Rozeboom (comps.): The psychology of knowing. Nueva York: Gordon \& Breach, 177-205.

MOWRER, O. H. (1960a). Learning theory and bebavior. Nueva York: Wiley.

MOWRER, O. H. (1960b). Learning theory and the symbolic processes. Nueva York: Wiley.

NATSOULAS, T. (1967). What are perceptual reports about? Psychological Review, 67 (4), $1249-1272$.

Natsoulas, T. (1970). Concerning introspective "knowledge". Psychological Bulletin, 73 (2), 89-111.

NeISSER, U. (1979). Psicología cognoscitiva. México: Trillas (edic. origin., 1967).

NeIsser, U. (1981). Procesos cognitivos y realidad. Principios e implicaciones de la psicología cognitiva. Madrid: Marova (edic. origin., 1976).

NEWELL, A. (1973). You can't play twenty questions with nature and win. En M. Chese (comp.): Visual information processing. Nueva York: Academic Press.

Newell, A., y SimON, H. A. (1972). Human problem solving. Englewood Cliffs, N. J.: Prentice-Hall.

NISBETT, R. E., y WILSON, T. D. (1977). Telling more than we can know: verbal reports on mental processes. Psychological Review, 84 (3), 231-259.

NoRman, D. A., y RUMElHART, D. E. (1975). Explorations in cognition. San Francisco: Freeman.

ORNE, M. T. (1962). On the social psychology of the psychological experiment: With particular reference to demand characteristics and their implication. American Psychologist, 17, 776-783.

ORNE, M. T. (1969). Demand characteristics and the concept of quasi-controls. En R. Rosenthaly R. L. Rosnow (comps.): Artifact in behavioral research. Nueva York: Academic Press, 143-179.

PAIviO, A. (1971). Imaginery and verbal processes. Nueva York: Holt, Rinehart \& Winston.

Pelechano, V. (1979). Terapia y modificación de conducta. Boletín Informativo de la Fundación Juan March, 78, 3-18.

Peterson, L. R., y PETERSON, M. J. (1959). Short-term retention of individual verbal item. Journal of Experimental Psychology, 58, 193-198.

PInillos, J. L. (1980). Conductismo y psicología cognitiva. Revista de Historia de la Psicologia, 1 (3-4), 267-282.

POPPER, K. R. (1963). Conjectures and refutations. Londres: Routledge \& Kegan Paul.

POSNER, M. I. (1978). Chronometric explorations of mind. Hillsdale, N. J.: Lawrence Erlbaum Associates.

POSTMAN, L., y STARK, R. (1969). Role of response availability in transfer and interference. Journal of Experimental Psychology, 76, 168-177. 
Premack, D. C. (1984). El conocimiento animal. En M. T. Anguera y J. J. Veá (comps.): Conducta animal y representaciones mentales. Barcelona: PPU, 145-147 (orig. publicado en 1983).

RAJECKI, D. W. (comp.) (1983). Comparing Behavior: Studying Man studying Animals. Hillsdale, N. J.: Lawrence Erlbaum Associates.

RIVIĖRE, A. (1984). Sobre la multiplicidad de las representaciones. Un viaje para los vericuetos de los lenguajes del pensamiento. En J. Mayor (comp.) Actividad bumana y procesos cognitivos. Madrid: Alhambra, 109-129.

Rock, I. (1957). The role of repetition in associative learning. American Journal of Psycho$\log y, 70,186-193$.

RODRIGO, M. J. (1982). Las posibilidades del análisis de tareas como técnica para el estudio de los procesos mentales. Infancia y Aprendizaje, 19-20, 159-173.

RODRÍGUEZ DOMígueZ, S. (1984). Modificación cognitiva de conducta: ¿Alternativa o integración psicoterapéutica? Comunicación presentada en el Symposium sobre Actividad Humana y Procesos Cognitivo. Madrid, diciembre 1984.

RoITBLAT, H. L. (1984). El significado de la representación en la conducta animal. En M.T. Anguera y J. J. Veá (comps.): Conducta animal y representaciones mentales. Barcelona: PPU, 89-142 (orig. publicado en 1982).

ROSENTHAL, R. (1964). The effect of the experimenter on the results of psychological research. En B. A. Maher (comp.): Progress in Experimental Personality Research, vol. 1 : Nueva York: Academic Press, 79-114.

RuCH, P. (1963). Psychology and life. Chicago: Scott, Foresman \& Co.

SALDAÑA, C. Alternativas conductuales a problemas de salud. Anuario de Psicologia, 30/31 (1-2), 59-74.

SEOANE, J. (1979). Inteligencia artificial y procesamiento de la información. Boletín Informativo de la Fundación Juan March, 3-21.

SEOANE, J. (1980). Problemas epistemológicos de la psicología actual. Análisis y Modificación de Conducta, 6 (11-12), 91-107.

SeoAne, J. (1982). Psicologia cognitiva y psiçología del conocimiento. Boletín de Psicologia, 1, 27-41. [Reimpreso en Boletín de Psicologia, 1984, 1-2, 27-41.]

SKINNER, B. F. (1957). Verbal behavior. Nueva York: Appletonentury-Crofts.

SKINNER, B. F. (1963). Behaviorism at fifty. Science, 140, 915-958.

SKINNER, B. F. (1977). Why I am not a cognitive psychologist. Behaviorism, 5, 1-10.

SPENCE, K. W. (1937). The differential response in animals to stimuli varying within a single dimension. Psychological Review, 44, 430-444.

STADDON, J. E. R. (1984). El conocimiento en los animales: El aprendizaje como ensamblaje de programas. En M. T. Anguera y J. J. Veá (comps.): Conducta animal y representaciones mentales. Barcelona: PPU, 61-69 (orig. publicado en 1981).

STEIN, N. L., y TRABASSO, T. (1982). What's in a stiry: An approach to comprehension and instruction. En R. Glaser (comp.): Advances in instructional psychology. Hillsdale, N. J.: Lawrence Erlbaum Associates, vol. 2, 213-267.

STERNBERG, S. (1975). Memory scanning: New findings and current controversies. The Quarterly Journal of Experimental Psycbology, 27, 1-32.

STERNBERG, S. (1969a). The discovery of processing stages: Extensions of Donders' method. Acta Psychologica, 30, 276-315.

STERNBERG, S. (1969b). Memory-scanning: Mental processes revealed by reaction-time experiments. American Scientist, $57,421-457$.

Stevens, A. L., y Collins, A. (1982). Multiple conceptual models of a complex system. En R. E. Snow; P. A. Federico y W. E. Montague (comps.): Aptitude, learning, and instruction. Vol. 2: Cognitive processes analysis of learning and problem solving. Hillsdale, N. J.: Lawrence Erlbaum Associates, 177-197.

TAYLOR, D. A. (1976). Stage analysis of reaction time. Psychological Bulletin, 83, 161-191.

TOLMAN, E. C. (1932). Purposive behavior in animals and men. Nueva York: Appleton-Century-Crofts.

Tous, J. M. Psicología Experimental. Problemas de teoria y método. Barcelona: Omega.

VEÁ, J. J. (1983). Procesos cognitivos en la conducta animàl: Una aplicación psicolingüística. Anuario de Psicologia, 28(1), 59-83.

VEÁ, J. J. (1984). Conducta animal y representaciones mentales: Aspectos epistemológicos. En M. T. Anguera y J. J. Veá (comps.): Conducta animal y representaciones mentales. Barcelona: PPU, 73-86.

WANN, T. W. (comp.) (1964). Behaviorism and phenomenology: Contrasting bases for modern psychology. Chicago: University of Chicago Press.

Wason, P. C., y Evans, J. S. B. (1975). Dual processes in reasoning? Cognition, 3, 141-154.

WATSON, J. B. (1913). Psychology as the behaviorist views it. Psychological Review, 20, $158-177$.

WATSON, J. B. (1919). Psychology from the stadpoint of a behaviorist. Philadelphia: Lippincott.

WATSON, J. B. (1920). Is thinking merely the action of language mechanisms? British Journal of Psychology, 11, 100-103. 
WEIMER, W. B. (1979). Notes on the methodology of scientific research. Hillsdale, N. J.: Lawrence Erlbaum Associates.

WHITE, S. J. (1976). The active organism in theoretical behaviorism. Human Development, $19,99-107$.

WhITTAKER, J. O. (1965). Introduction to psychology. Philadelphia: Saunders.

WICKELGREN, W. (1979). Cognitive psychology. Englewood Cliffs, N. J.: Prentice-Hall.

WILSON, G. T. (1978). Cognitive behavior therapy: Paradigm shift or passing phase. En J. J. Foreyt y D. P. Rathjen (comps.): Cognitive behavior therapy: Research and application. Nueva York: Plenum Press.

WILSON, K. W. (1980). From associations to structure. The course of cognition. Amsterdam: North-Holland.

WOLPE, J. (1958). Psychotherapy by reciprocal inhibition. Stanford: Stanford University Press.

WOLPE, J. (1969). The practice of behavior therapy. Nueva York: Pergamon Press.

WOLPE, J. (1978). Cognition and causation in human behavior and its therapy. American Psychologist, 33, 437-446.

WolPE, J., y LAZARUS, A. A. (1966). Behavior therapy techniques. Nueva York: Pergamon Press.

YATES, A. J. (1976). Terapia del comportamiento. México: Trillas [edic. origin., 1970].

YELA, M. (1974). La estructura de la conducta. Estimulo, situación y conciencia. Madrid: Real Academia de Ciencias Morales y Políticas.

Yela, M. La evolución del conductismo. Análisis y Modificación de Conducta, 6 (11-12), 147-179.

ZacCagnini, J. L., y Delclaux, I. (comps.): Psicología cognitiva y procesamiento de la información. En I. Delclaux y J. Seoane (comps.): Psicologia cognitiva y procesamiento de la información. Madrid: Pirámide, 39-62.

ZEIGLER, B. P.; ElZAS, M. S.; KLIR, G. J., y ÖREN, T. I. (comps.): Methodology in systems modelling and simulation. Amsterdam: North-Holland. 